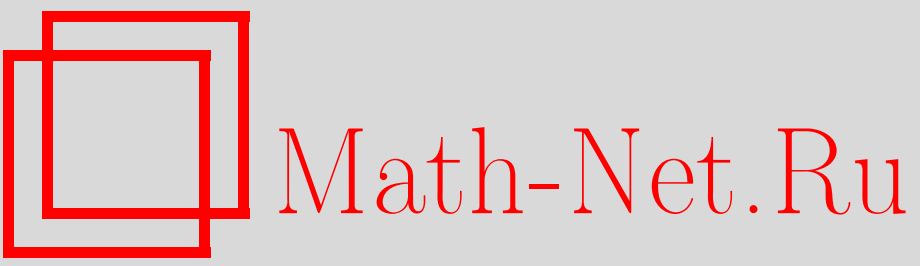

В. Р. Гаврилов, В. Н. Мельников, $D$-мерные $p$-бранные космологические модели, ассоциированные с алгебрами Ли типа $A_{m}, T M \Phi, 2000$, том 123, номер 3, 374-394

DOI: https://doi.org/10.4213/tmf610

Использование Общероссийского математического портала Math-Net.Ru подразумевает, что вы прочитали и согласны с пользовательским соглашением

http://www.mathnet.ru/rus/agreement

Параметры загрузки:

IP : 35.173 .137 .237

26 апреля 2023 г., 17:59:49 
ТЕОРЕТИЧЕСКАЯ

И МАТЕМАТИЧЕСКАЯ

ФИЗИКА

Том 123, № 3

июнь, 2000

(C) 2000 r.

В.Р. Гаврилов* , В.Н. Мельников*

\section{$D$-МЕРНЫЕ $p$-БРАННЫЕ КОСМОЛОГИЧЕСКИЕ МОДЕЛИ, АССОЦИИРОВАННЫЕ С АЛГЕБРАМИ ЛИ ТИПА $A_{m}$}

Изучена $D$-мерная космологическая модель на многообразии $\mathbf{M}=\mathbb{R} \times M_{0} \times \cdots$ $\cdots \times M_{n}$, описывающая эволюцию $n+1$ эйнштейновских фактор-пространств $M_{i}$ в теории с несколькими дилатонными скалярными полями и дифференциальными формами, допускающими интерпретацию в терминах пересекающихся $p$-бран. Уравнения движения модели сведены к уравнениям Эйлера-Лагранжа так называемой псевдоевклидовой системы типа цепочки Тоды. Предполагается, что характеристические векторы, которые связаны с конфигурацией $p$-бран и константами их связи с дилатонными скалярными полями, могут быть интерпретированы как корни алгебры Ли типа $A_{m} \equiv s l(m+1, \mathbb{C})$. В этом случае модель сводится к открытой цепочке Тоды и интегрируется известными методами. Результирующая метрика представлена в форме типа решения Казнера. Выделена частная модель, описывающая эволюцию типа фридмановской трехмерного внешнего пространства $M_{0}$ (в эйнштейновской конформной калибровке) и сжатие внутренних пространств $M_{1}, \ldots, M_{n}$.

\section{1. ВВЕДЕНИЕ}

В последние годы наблюдается возросший интерес к классическим $p$-бранным решениям в теориях супергравитации различных размерностей [1-19]. Этот интерес вызван предположением, что $D=11$ супергравитация является низкоэнергетическим эффективным полевым пределом 11-мерной фундаментальной $M$-теории, которая (вместе с так называемой $F$-теорией) является кандидатом для объединения пяти известных 10-мерных суперструнных моделей [20-27]. Классические $p$-бранные решения можно рассматривать как метод исследования взаимосвязей суперструнных моделей и $M$-теории.

В данной работе мы рассматриваем обобшенный бозонный сектор (без членов Черна-Саймонса) ранних теорий супергравитации [28] в форме многомерной гравитационной модели с несколькими дилатонными скалярными полями и дифференциальными формами различных рангов, допускающими интерпретацию в терминах пересекающихся $p$-бран. Как было показано в [17], для космологических и статических сферичес-

Центр гравитации и фундаментальной метрологии ВНИИМС, Москва, Россия. Е-mail: melnikov@rgs.phys.msu.su 
ки-симметричных пространственно-временных многообразий уравнения движения такой модели сводятся к уравнениям Эйлера-Лагранжа так называемой псевдоевклидовой лагранжевой системы типа цепочки Тоды. Этот результат воспроизведен в разделе 2. Методы интегрирования псевдоевклидовых систем типа цепочки Тоды развиты в работах [29-36], посвященных многомерной космологии с многокомпонентной идеальной жидкостью. Эти методы основаны на геометрии типа геометрии Минковского для характеристических векторов, определяющих потенциал псевдоевклидовой системы типа цепочки Тоды. Если характеристические векторы образуют ортогональную систему, то псевдоевклидова система типа цепочки Тоды интегрируема. Соответствующие $p$-бранные модели изучены в работах $[10,12,17]$. В данной работе мы применяем эти методы для интегрирования $p$-бранной модели, сводимой к открытой цепочке Тоды. Характеристические векторы такой модели могут быть интерпретированы как корни алгебры Ли типа $A_{m} \equiv s l(m+1, \mathbb{C})$ по классификации Картана. В разделе 3 мы интегрируем $p$-бранную модель и представляем результирующую метрику в форме типа решения Казнера, используя для решения уравнений движения цепочки Тоды элегантную методику, предложенную Андерсон [37]. В разделе 4 исследована метрика, полученная для некоторой частной $p$-бранной модели, ассоциированной с алгеброй Ли $A_{2}$. Эта метрика описывает эволюцию типа фрридмановской трехмерного внешнего пространства (в эйнштейновской конформной калибровке) и сжатие внутренних пространств.

\section{2. ОБШАЯ МОДЕЛЬ}

Следуя работам $[4,5,9-14,17-19]$, мы рассматриваем классическую модель теории гравитации с несколькими дилатонными скалярными полями $\varphi^{\alpha}$ и дифференциальными $n_{a}$-формами $F_{M_{1} \ldots M_{n_{a}}}^{a}$ в (псевдо)римановом пространственно-временном многообразии М размерности $D$. Действие модели имеет вид

$$
S=\int_{\mathbf{M}} d^{D} z \sqrt{|g|}\left(R[g]-\sum_{\alpha, \beta=1}^{l} h_{\alpha \beta} g^{M N} \partial_{M} \varphi^{\alpha} \partial_{N} \varphi^{\beta}-\sum_{a \in \Delta} \frac{1}{n_{a} !} \mathrm{e}^{2 \lambda_{a}(\varphi)}\left(F^{a}\right)^{2}\right)
$$

где $g=g_{M N} d z^{M} \otimes d z^{N}$ - метрика с лоренцевой сигнатурой на многообразии $\mathbf{M}(M, N=$ $0,1, \ldots, D-1),|g|=\left|\operatorname{det}\left(g_{M N}\right)\right|,\left(h_{\alpha \beta}\right)$ - симметричная положительно определенная $(l \times l)$-матрица, $\lambda_{a}(\varphi)$ - линейная комбинация скалярных полей, т.е.

$$
\lambda_{a}(\varphi)=\sum_{\alpha=1}^{l} \lambda_{a, \alpha} \varphi^{\alpha}
$$

где $\lambda_{a, \alpha}-$ константы связи. Кроме того,

$$
\begin{aligned}
F^{a} & =\frac{1}{n_{a} !} F_{M_{1} \ldots M_{n_{a}}}^{a} d z^{M_{1}} \wedge \cdots \wedge d z^{M_{n_{a}}}=d A^{a} \\
A^{a} & =\frac{1}{\left(n_{a}-1\right) !} A_{M_{1} \ldots M_{n_{a}-1}}^{a} d z^{M_{1}} \wedge \cdots \wedge d z^{M_{n_{a}-1}}, \\
\left(F^{a}\right)^{2} & =F_{M_{1} \ldots M_{n_{a}}}^{a} F_{N_{1} \ldots N_{n_{a}}}^{a} g^{M_{1} N_{1}} \ldots g^{M_{n_{a}} N_{n_{a}}}
\end{aligned}
$$


Поле $A_{M_{1} \ldots M_{n_{a}-1}}^{a}$ может быть названо калибровочным потенциалом, соответствующим полю напряженности $F_{M_{1} \ldots M_{n_{a}}}^{a}$. Символом $\Delta$ обозначено некоторое конечное множество.

Действие (2.1) приводит к следующим уравнениям движения:

$$
\begin{gathered}
R_{M N}-\frac{1}{2} g_{M N} R=T_{M N}, \\
\triangle[g] \varphi^{\alpha}=\sum_{a \in \Delta} \frac{1}{n_{a} !} \lambda_{a}^{\alpha} \mathrm{e}^{2 \lambda_{a}(\varphi)}\left(F^{a}\right)^{2}, \quad \alpha=1, \ldots, l, \\
\nabla_{M_{1}}[g]\left(\mathrm{e}^{2 \lambda_{a}(\varphi)} F^{a, M_{1} \ldots M_{n_{a}}}\right)=0, \quad a \in \Delta .
\end{gathered}
$$

Правая часть уравнений Эйнштейна (2.6) имеет вид

$$
T_{M N}=T_{M N}[\varphi]+\sum_{a \in \Delta} \mathrm{e}^{2 \lambda_{a}(\varphi)} T_{M N}\left[F^{a}\right]
$$

где обозначено

$$
\begin{aligned}
T_{M N}[\varphi] & =\sum_{\alpha, \beta=1}^{l} h_{\alpha \beta}\left(\partial_{M} \varphi^{\alpha} \partial_{N} \varphi^{\beta}-\frac{1}{2} g_{M N} \partial_{P} \varphi^{\alpha} \partial^{P} \varphi^{\beta}\right), \\
T_{M N}\left[F^{a}\right] & =\frac{1}{n_{a} !}\left(-\frac{1}{2} g_{M N}\left(F^{a}\right)^{2}+n_{a} F_{M M_{2} \ldots M_{n_{a}}}^{a} F_{N}^{a, M_{2} \ldots M_{n_{a}}}\right) .
\end{aligned}
$$

В уравнениях (2.7), (2.8) оператор Лапласа-Бельтрами и ковариантная производная по отношению к метрике $g$ обозначены через $\triangle[g]$ и $\nabla_{M}[g]$, соответственно. Постоянные $\lambda_{a}^{\alpha}$ в (2.7) введены следуюшим образом:

$$
\lambda_{a}^{\alpha}=\sum_{\beta=1}^{l} h^{\alpha \beta} \lambda_{a, \beta},
$$

где $\left(h^{\alpha \beta}\right)$ - обратная матрица к $\left(h_{\alpha \beta}\right)$.

Рассмотрим модель при следующих предположениях. Пусть $D$-мерное пространство-время $\mathbf{M}$ разлагается в прямое произведение оси времени $\mathbb{R}$ и $n+1$ фактор-пространств $M_{0}, \ldots, M_{n}$, т.е.

$$
\mathbf{M}=\mathbb{R} \times M_{0} \times \cdots \times M_{n}
$$

Пусть

$$
g=-\mathrm{e}^{2 \gamma(t)} d t \otimes d t+\sum_{i=0}^{n} \mathrm{e}^{2 x^{i}(t)} g^{(i)}
$$

- метрика на М. Функция $\gamma(t)$ определяет калибровку времени, $g^{(i)}=g_{m_{i} n_{i}}^{(i)}\left(y_{(i)}\right) d y_{(i)}^{m_{i}} \otimes$ $d y_{(i)}^{n_{i}}$ - метрика с евклидовой сигнатурой на $M_{i}$. Предполагается, что $\left(M_{i}, g^{i}\right)$ является пространством Эйнштейна размерности $d_{i}$, т.е.

$$
R_{k_{i} l_{i}}\left[g^{(i)}\right]=\xi_{i} g_{k_{i} l_{i}}^{(i)}, \quad k_{i}, l_{i}=1, \ldots, d_{i} \equiv \operatorname{dim} M_{i}, \quad i=0, \ldots, n
$$


где $\xi_{i}$ - постоянные. В частном случае, когда $M_{i}$ является пространством постоянной римановой кривизны $K_{i}$, постоянная $\xi_{i}$ имеет вид $\xi_{i}=K_{i}\left(d_{i}-1\right), d_{i}>1$. Координаты на $\mathbf{M}$ расшепляются на интервалы

$$
\left(z^{0}, z^{1}, \ldots, z^{d_{0}}, \ldots, z^{D-d_{n}}, \ldots, z^{D-1}\right)=\left(t, y_{(0)}^{1}, \ldots, y_{(0)}^{d_{0}}, \ldots, y_{(n)}^{1}, \ldots, y_{(n)}^{d_{n}}\right) .
$$

Кроме того, предполагается, что скалярные поля $\varphi^{\alpha}$ и калибровочные потенциалы $A_{M_{1} \ldots M_{n_{a}-1}}^{a}$ зависят только от времени $t$.

Следуя основным идеям теории $p$-бран [1] и учитывая топологию (2.13) пространства-времени $\mathbf{M}$, построим калибровочные потенциалы $A_{M_{1} \ldots M_{n_{a}-1}}^{a}$ следующим образом. Предполагая, что каждое фактор-пространство $M_{i}$ является ориентируемым и связным, введем $d_{i}$-форму объема $M_{i}$

$$
\tau_{i}=\frac{1}{d_{i} !} \sqrt{\left|g^{(i)}\left(y_{(i)}\right)\right|} \delta_{M_{1}, \ldots, M_{d_{i}}}^{N(i)+1, \ldots, N(i)+d_{i}} d z^{M_{1}} \wedge \cdots \wedge d z^{M_{d_{i}}}
$$

где $N(i)=d_{0}+\cdots+d_{i-1}, N(0)=0$ и

$\delta_{\beta_{1}, \ldots, \beta_{k}}^{\alpha_{1}, \ldots, \alpha_{k}}=\left\{\begin{array}{l}1, \quad \text { если } \beta_{1}, \ldots, \beta_{k} \text { - четная перестановка символов } \alpha_{1}, \ldots, \alpha_{k}, \\ -1, \quad \text { если } \beta_{1}, \ldots, \beta_{k} \text { - нечетная перестановка символов } \alpha_{1}, \ldots, \alpha_{k}, \\ 0, \quad \text { если } \beta_{1}, \ldots, \beta_{k} \text { не является перестановкой символов } \alpha_{1}, \ldots, \alpha_{k} .\end{array}\right.$

Определим множество

$$
\Omega_{0}=\{\{0\},\{1\}, \ldots,\{n\},\{0,1\}, \ldots,\{0,1, \ldots, n\}\}
$$

всех упорядоченных совокупностей вида

$$
I=\left\{i_{1}, \ldots, i_{k}\right\} \in \Omega_{0}, \quad i_{1}<\cdots<i_{k} .
$$

Положим по определению

$$
I_{0} \equiv\{0, \ldots, n\}, \quad \bar{I} \equiv I_{0} \backslash I .
$$

Каждой совокупности $I=\left\{i_{1}, \ldots, i_{k}\right\} \in \Omega_{0}$ поставим в соответствие подмногообразие

$$
M_{I} \equiv M_{i_{1}} \times \cdots \times M_{i_{k}}
$$

размерности

$$
\operatorname{dim} M_{I} \equiv d(I)=d_{i_{1}}+\cdots+d_{i_{k}}
$$

с формой объема

$$
\tau(I)=\tau_{i_{1}} \wedge \cdots \wedge \tau_{i_{k}} .
$$

В соответствии с терминологией теории $p$-бран [1] $\left(n_{a}-1\right)$-форма потенциала

$$
A^{(a, e, I)}=\Phi^{(a, e, I)}(t) \tau(I), \quad \operatorname{rank} A^{(a, e, I)} \equiv n_{a}-1=d(I), \quad a \in \Delta,
$$


где $\Phi^{(a, e, I)}(t)$ - скалярная функция, описывает электрически заряженную $p$-брану $(p=$ $\left.n_{a}-2\right)$, вложенную в многообразие $M_{I}$, которое называется мировым объемом этой $p$-браны. Подмногообразие $\mathbb{R} \times M_{\bar{I}}$ называется трансверсальным для этой $p$-браны. Поле напряженности $n_{a}$-формы, соответствуюшей форме $(2.24)$ калибровочного потенциала, имеет вид

$$
F^{(a, e, I)}=d \Phi^{(a, e, I)}(t) \wedge \tau(I)
$$

Поле напряженности $n_{b}$-формы

$$
\begin{gathered}
F^{(b, m, J)}=\mathrm{e}^{-2 \lambda_{b}(\varphi)} *\left(d \Phi^{(b, m, J)}(t) \wedge \tau(J)\right), \\
\operatorname{rank} F^{(b, m, J)} \equiv n_{b}=d(\bar{J}), \quad b \in \Delta,
\end{gathered}
$$

описывает $p$-брану $(p=d(\bar{J})-1)$ с зарядом магнитного типа. Подмногообразие $M_{\bar{J}}$ является мировым объемом этой $p$-браны. Символом $*$ обозначен оператор Ходжа на многообразии $(\mathbf{M}, g)$, т.е.

$$
(* F)_{M_{1} \ldots M_{D-k}}=\frac{\sqrt{|\operatorname{det} g|}}{k !} \varepsilon_{N_{1} \ldots N_{k} M_{1} \ldots M_{D-k}} F^{N_{1} \ldots N_{k}} .
$$

Две $p$-браны с мировыми объемами $M_{I}$ и $M_{J}$ называются пересекаюшимися, если $I \cap J \neq \varnothing$.

В данной работе рассматриваются так называемые композитные $p$-браны [2], т.е. по определению полагается

$$
F^{a}=\sum_{I \in \Omega_{a, e}} F^{(a, e, I)}+\sum_{J \in \Omega_{a, m}} F^{(a, m, J)}
$$

где $\Omega_{a, e} \subset \Omega_{0} \sqcup\{\varnothing\}$ - подмножество (возможно пустое) всех совокупностей $I \in \Omega_{0}$ таких, что $n_{a} \equiv \operatorname{rank} F^{(a, e, I)}=d(I)+1 ; \Omega_{a, m} \subset \Omega_{0} \sqcup\{\varnothing\}-$ подмножество (возможно пустое) всех совокупностей $J \in \Omega_{0}$ таких, что $n_{a} \equiv \operatorname{rank} F^{(a, m, J)}=d(\bar{J})=D-1-d(J)$. Очевидно, что $\Omega_{a, m}=\varnothing$ для $n_{a}=D-1, D$.

Используя предположения (2.15), мы получаем следующие ненулевые компоненты тензора Риччи для метрики (2.14):

$$
\begin{aligned}
R_{0}^{0} & =\mathrm{e}^{-2 \gamma}\left(\sum_{i=0}^{n} d_{i}\left(\dot{x}^{i}\right)^{2}+\ddot{\gamma_{0}}-\dot{\gamma} \dot{\gamma_{0}}\right) \\
R_{n_{i}}^{m_{i}} & =\left\{\xi_{i} \mathrm{e}^{-2 x^{i}}+\left[\ddot{x}^{i}+\dot{x}^{i}\left(\dot{\gamma}_{0}-\dot{\gamma}\right)\right] \mathrm{e}^{-2 \gamma}\right\} \delta_{n_{i}}^{m_{i}},
\end{aligned}
$$

где обозначено

$$
\gamma_{0}=\sum_{i=0}^{n} d_{i} x^{i}
$$

Индексы $m_{i}$ и $n_{i}$ в $(2.30)$ для $i=0, \ldots, n$ принимают все значения от $D-\sum_{j=i}^{n} d_{j}$ до $D-\sum_{j=i}^{n} d_{j}+d_{i}-1\left(D=1+\sum_{i=0}^{n} d_{i}=\operatorname{dim} \mathbf{M}\right)$. 
При сделанных предположениях относительно $F^{a}$-полей уравнения типа максвелловских (2.8) и тождества Бианки $d F^{a}=0$ имеют соответственно вид

$$
\begin{gathered}
\frac{d}{d t}\left[\exp \left\{\gamma_{0}-\gamma-2 \sigma(I)+2 \lambda_{a}(\phi)\right\} \dot{\Phi}^{(a, e, I)}(t)\right]=0, \quad I \in \Omega_{a, e}, \quad a \in \Delta, \\
\frac{d}{d t}\left[\exp \left\{\gamma_{0}-\gamma-2 \sigma(J)-2 \lambda_{a}(\phi)\right\} \dot{\Phi}^{(a, m, J)}(t)\right]=0, \quad J \in \Omega_{a, m}, \quad a \in \Delta,
\end{gathered}
$$

где

$$
\sigma(I)=\sum_{i \in I} d_{i} x^{i}
$$

Интегрируя (2.32) и (2.33), получаем

$$
\begin{aligned}
\dot{\Phi}^{(a, e, I)}(t) & =Q_{(a, e, I)} \exp \left\{\gamma-\gamma_{0}+2 \sigma(I)-2 \lambda_{a}(\phi)\right\}, \quad I \in \Omega_{a, e}, \quad a \in \Delta, \\
\dot{\Phi}^{(a, m, J)}(t) & =Q_{(a, m, J)} \exp \left\{\gamma-\gamma_{0}+2 \sigma(J)+2 \lambda_{a}(\phi)\right\}, \quad J \in \Omega_{a, m}, \quad a \in \Delta
\end{aligned}
$$

где $Q_{(a, e, I)}$ и $Q_{(a, m, J)}-$ произвольные постоянные.

Для того чтобы получить тензоры $T\left[F^{a}\right]_{N}^{M}$ в блок-диагональном виде, наложим следующие ограничения [17].

1. Отсутствуют элементы $I, J \in \Omega_{a, e}$ или $I, J \in \Omega_{a, m}, a \in \Delta$, такие, что

$$
I=(I \cap J) \sqcup\{i\}, \quad J=(I \cap J) \sqcup\{j\}, \quad i \neq j, \quad d_{i}=d_{j}=1 .
$$

Здесь пересечение $I \cap J$ может быть пустым.

2. Отсутствуют элементы $I \in \Omega_{a, e}$ и $J \in \Omega_{a, m}, a \in \Delta$, такие, что

$$
\bar{J}=I \sqcup\{i\}, \quad d_{i}=1 .
$$

Итак, тензор энергии-импульса $F^{a}$-поля, $a \in \Delta$, имеет блок-диагональный вид, если справедливы ограничения (2.37), (2.38). Используя (2.35), (2.36), представим его ненулевые компоненты в виде

$$
\begin{aligned}
\mathrm{e}^{2 \lambda_{a}(\phi)} T\left[F^{a}\right]_{0}^{0}= & -\frac{1}{2} \mathrm{e}^{-2 \gamma_{0}}\left(\sum_{I \in \Omega_{a, e}} Q_{(a, e, I)}^{2} \mathrm{e}^{2 \sigma(I)-2 \lambda_{a}(\phi)}+\right. \\
& +\sum_{J \in \Omega_{a, m}} Q_{(a, m, J)}^{2} \mathrm{e}^{\left.2 \sigma(J)+2 \lambda_{a}(\phi)\right)}, \\
\mathrm{e}^{2 \lambda_{a}(\phi)} T\left[F^{a}\right]_{n_{i}}^{m_{i}}= & -\frac{1}{2} \mathrm{e}^{-2 \gamma_{0}}\left(\sum_{I \in \Omega_{a, e}}\left[2 \delta_{i I}-1\right] Q_{(a, e, I)}^{2} \mathrm{e}^{2 \sigma(I)-2 \lambda_{a}(\phi)}+\right. \\
& \left.+\sum_{J \in \Omega_{a, m}}\left[2 \delta_{i J}-1\right] Q_{(a, m, J)}^{2} \mathrm{e}^{\left.2 \sigma(J)+2 \lambda_{a}(\phi)\right)}\right) \delta_{n_{j}}^{m_{i}},
\end{aligned}
$$

где

$$
\delta_{i I}=\sum_{j \in I} \delta_{i j}
$$


При сделанных предположениях тензор энергии-импульса дилатонных скалярных полей имеет вид

$$
\left(T[\varphi]_{N}^{M}\right)=\frac{1}{2} \mathrm{e}^{-2 \gamma}\left(\sum_{\alpha, \beta=1}^{l} h_{\alpha \beta} \dot{\varphi}^{\alpha} \dot{\varphi}^{\beta}\right) \operatorname{diag}(-1,1, \ldots, 1) .
$$

Уравнения Эйнштейна (2.6) могут быть записаны как $R_{N}^{M}=T_{N}^{M}-T \delta_{N}^{M} /(D-2)$. Далее использованы уравнения

$$
R_{0}^{0}-\frac{R}{2}=T_{0}^{0}, \quad R_{n_{i}}^{m_{i}}=T_{n_{i}}^{m_{i}}-\frac{T \delta_{n_{i}}^{m_{i}}}{D-2} .
$$

Применяя (2.29), (2.30), (2.39), (2.40), (2.42), мы получаем эти уравнения в виде

$$
\begin{gathered}
\frac{1}{2}\left(\sum_{i, j=0}^{n} G_{i j} \dot{x}^{i} \dot{x}^{j}+\sum_{\alpha, \beta=1}^{l} h_{\alpha \beta} \dot{\varphi}^{\alpha} \dot{\varphi}^{\beta}\right)+V=0 \\
\ddot{x}^{i}+\left(\dot{\gamma}_{0}-\dot{\gamma}\right) \dot{x}^{i}=\mathrm{e}^{2 \gamma}\left\{-\xi^{i} \mathrm{e}^{-2 x^{i}}-\right. \\
\quad-\sum_{a \in \Delta}\left[\sum_{I \in \Omega_{a, e}}\left(\delta_{i I}-\frac{d(I)}{D-2}\right) Q_{(a, e, I)}^{2} \mathrm{e}^{2 \sigma(I)-2 \lambda_{a}(\phi)}+\right. \\
\left.\left.+\sum_{J \in \Omega_{a, m}}\left(\delta_{i J}-\frac{d(J)}{D-2}\right) Q_{(a, m, J)}^{2} \mathrm{e}^{2 \sigma(J)+2 \lambda_{a}(\phi)}\right] \mathrm{e}^{-2 \gamma_{0}}\right\}
\end{gathered}
$$

где обозначено

$$
\begin{gathered}
G_{i j}=d_{i} \delta_{i j}-d_{i} d_{j} \\
V=\frac{1}{2} \mathrm{e}^{2 \gamma}\left[\sum _ { a \in \Delta } \mathrm { e } ^ { - 2 \gamma _ { 0 } } \left(\sum_{I \in \Omega_{a, e}} Q_{(a, e, I)}^{2} \mathrm{e}^{2 \sigma(I)-2 \lambda_{a}(\phi)}+\right.\right. \\
\left.\left.+\sum_{J \in \Omega_{a, m}} Q_{(a, m, J)}^{2} \mathrm{e}^{2 \sigma(J)+2 \lambda_{a}(\phi)}\right)-\sum_{i=0}^{n} \xi^{i} d_{i} \mathrm{e}^{-2 x^{i}}\right] .
\end{gathered}
$$

При сделанных предположениях уравнения (2.7) принимают вид

$$
\begin{aligned}
\ddot{\varphi}^{\alpha}+\left(\dot{\gamma}_{0}-\dot{\gamma}\right) \dot{\varphi}^{\alpha}= & \mathrm{e}^{2 \gamma-2 \gamma_{0}} \sum_{a \in \Delta} \lambda_{a}^{\alpha}\left(\sum_{I \in \Omega_{a, e}} Q_{(a, e, I)}^{2} \mathrm{e}^{2 \sigma(I)-2 \lambda_{a}(\phi)}-\right. \\
& \left.-\sum_{J \in \Omega_{a, m}} Q_{(a, m, J)}^{2} \mathrm{e}^{2 \sigma(J)+2 \lambda_{a}(\phi)}\right)
\end{aligned}
$$

Нетрудно убедиться в том, что уравнения $(2.43),(2.44),(2.47)$ могут быть представлены как уравнения Эйлера-Лагранжа, полученные из лагранжиана

$$
L=\mathrm{e}^{\gamma_{0}-\gamma}\left[\frac{1}{2}\left(\sum_{i, j=0}^{n} G_{i j} \dot{x}^{i} \dot{x}^{j}+\sum_{\alpha, \beta=1}^{l} h_{\alpha \beta} \dot{\varphi}^{\alpha} \dot{\varphi}^{\beta}\right)-V\right]
$$


рассматриваемого как функция обобщенных координат $\gamma, x^{i}, \varphi^{\alpha}$. Уравнение $\partial L / \partial \gamma=$ $d(\partial L / \partial \dot{\gamma}) / d t$ приводит к условию нулевой энергии (2.43). После фиксации калибровки $\gamma=F\left(x^{i}, \varphi^{\alpha}\right)$ уравнения $(2.44),(2.47)$ можно рассматривать как уравнения Эйлера-Лагранжа, полученные из лагранжиана (2.48) при условии связи (2.43). Далее мы используем так называемую гармоническую калибровку времени

$$
\gamma \equiv \gamma_{0}=\sigma\left(I_{0}\right)=\sum_{i=0}^{n} d_{i} x^{i}
$$

Легко проверить, что временна́я координата $t$ является гармонической функцией в этой калибровке, т.е. $\triangle[g] t=0$.

Введем в рассмотрение $(n+l+1)$-мерное вешественное векторное пространство $\mathbb{R}^{n+l+1}$. Обозначим через $e_{A}, A=0,1, \ldots, n+l$, канонический базис в $\mathbb{R}^{n+l+1}\left(e_{1}=\right.$ $(1,0, \ldots, 0)$ и т.д.). Определим следуюшие векторы:

1. Вектор, координаты которого нужно найти,

$$
x(t)=\sum_{A=0}^{n+l} x^{A}(t) e_{A}, \quad\left(x^{A}(t)\right)=\left(x^{0}(t), \ldots, x^{n}(t), \varphi^{1}(t), \ldots, \varphi^{l}(t)\right) .
$$

2. Вектор, соответствуюший фактор-пространству $M_{k}$ с ненулевым тензором Риччи. Далее мы называем его вектором, индуцированным кривизной пространства $M_{k}$,

$$
V_{k}=\sum_{A=0}^{n+l} V_{k}^{A} e_{A}=-\frac{2}{d_{k}} e_{k}, \quad\left(V_{k}^{A}\right)=-2\left(\frac{\delta_{k}^{i}}{d_{k}}, 0, \ldots, 0\right) .
$$

3. Вектор, индуцированный электрически заряженной $p$-браной $\left(p=n_{a}-2=\right.$ $d(I)-1)$ с мировым объемом $M_{I}$,

$$
U_{(a, e, I)}=\sum_{A=0}^{n+l} U_{(a, e, I)}^{A} e_{A}, \quad\left(U_{(a, e, I)}^{A}\right)=2\left(\delta_{i I}-\frac{d(I)}{D-2},-\lambda_{a}^{\alpha}\right)
$$

4. Вектор, индуцированный $p$-браной $(p=d(\bar{J})-1)$ с мировым объемом $M_{\bar{J}}$, обладающей магнитным зарядом,

$$
U_{(a, m, J)}=\sum_{A=0}^{n+l} U_{(a, m, J)}^{A} e_{A}, \quad\left(U_{(a, m, J)}^{A}\right)=2\left(\delta_{i J}-\frac{d(J)}{D-2}, \lambda_{a}^{\alpha}\right)
$$

Система векторов $V_{k}, U_{(a, e, I)}$ и $U_{(a, m, J)}$ характеризует пространство-время $\mathbf{M}$, конфигурацию $p$-бран и константы их связи с дилатонными скалярными полями. Далее эти векторы называются характеристическими.

Пусть $\langle\cdot, \cdot\rangle$ - симметричная билинейная форма на $\mathbb{R}^{n+l+1}$ такая, что

$$
\left\langle e_{A}, e_{B}\right\rangle=\bar{G}_{A B},
$$


где по определению

$$
\left(\bar{G}_{A B}\right)=\left(\begin{array}{cc}
G_{i j} & 0 \\
0 & h_{\alpha \beta}
\end{array}\right)
$$

Эта форма является невырожденной, обратная матрица к $\left(G_{A B}\right)$ записывается следующим образом:

$$
\left(\bar{G}^{A B}\right)=\left(\begin{array}{cc}
\frac{\delta^{i j}}{d_{i}}+\frac{1}{2-D} & 0 \\
0 & h^{\alpha \beta}
\end{array}\right) .
$$

Форма $\langle\cdot, \cdot\rangle$ наделяет пространство $\mathbb{R}^{n+l+1}$ метрикой, сигнатура которой имеет вид $(-,+, \ldots,+)[31]$. Обычным образом можно ввести ковариантные компоненты векторов. Для характеристических векторов $V_{k}, U_{(a, e, I)}, U_{(a, m, J)}$ ковариантные компоненты имеют вид

$$
\begin{aligned}
V_{k, A} & =2\left(d_{i}-\delta_{k i}, 0, \ldots, 0\right), \\
U_{(a, e, I), A} & =2\left(d_{i} \delta_{i I},-\lambda_{a, \alpha}\right), \\
U_{(a, m, J), A} & =2\left(d_{i} \delta_{i J}, \lambda_{a, \alpha}\right) .
\end{aligned}
$$

Значения билинейной формы $\langle\cdot, \cdot\rangle$ для $V_{k}, U_{(a, e, I)}, U_{(a, m, J)}$ записываются следуюшим образом:

$$
\begin{aligned}
\left\langle V_{i}, V_{k}\right\rangle & =4\left(\frac{\delta_{i k}}{d_{k}}-1\right), \\
\left\langle V_{k}, U_{(a, e, I)}\right\rangle & =\left\langle V_{k}, U_{(a, m, I)}\right\rangle=-4 \delta_{k I}, \\
\left\langle U_{(a, e, I)}, U_{(b, e, J)}\right\rangle & =\left\langle U_{(a, m, I)}, U_{(b, m, J)}\right\rangle= \\
& =4\left[d(I \cap J)-\frac{d(I) d(J)}{D-2}+\sum_{\alpha, \beta=1}^{l} h_{\alpha \beta} \lambda_{a}^{\alpha} \lambda_{b}^{\beta}\right], \\
\left\langle U_{(a, e, I)}, U_{(b, m, J)}\right\rangle & =4\left[d(I \cap J)-\frac{d(I) d(J)}{D-2}-\sum_{\alpha, \beta=1}^{l} h_{\alpha \beta} \lambda_{a}^{\alpha} \lambda_{b}^{\beta}\right] .
\end{aligned}
$$

Вектор $y \in \mathbb{R}^{n+l+1}$ называется времениподобным, пространственноподобным или изотропным, если $\langle y, y\rangle$ имеет отрицательное, положительное или нулевое значения, соответственно. Векторы $y$ и z называются ортогональными, если $\langle y, z\rangle=0$. Заметим, что индуцированный кривизной вектор $V_{k}$ всегда является времениподобным, в то время как вектор $U_{(a, v, I)}(v=e, m)$, индуцированный $p$-браной, допускает произвольное значение $\left\langle U_{(a, v, I)}, U_{(a, v, I)}\right\rangle$.

Для обозначения векторов, индуцированных $p$-бранами, а также соответствующих $F^{a}$-полей и их потенциалов, удобно использовать следующий общий индекс:

$$
s=(a, v, I), \quad I \in \Omega_{a, v}, \quad v=e, m, \quad a \in \Delta .
$$

Обозначим через $S$ множество всевозможных элементов $s$, т.е.

$$
S=\bigsqcup_{v=e, m}\left(\bigsqcup_{a \in \Delta}\{a\} \times\{v\} \times\left\{\Omega_{a, v}\right\}\right) .
$$


Пусть $S_{*} \subset S$ - подмножество всех $s \in S$ таких, что $Q_{s} \neq 0$. Определим биекцию $f: S_{*} \cong\left\{1,2, \ldots,\left|S_{*}\right|\right\}$. Обозначим натуральное число $f(s)$, соответствуюшее $s$, той же буквой $s$.

Используя обозначение $\langle\cdot, \cdot\rangle$ и векторы $(2.50)-(2.53)$, можно записать лагранжиан (2.48) и условие нулевой энергии (2.43) в гармонической калибровке времени следующим образом:

$$
L=\frac{1}{2}\langle\dot{x}, \dot{x}\rangle-V, \quad E=\frac{1}{2}\langle\dot{x}, \dot{x}\rangle+V \equiv 0,
$$

где потенциал $V$ имеет вид

$$
V=\sum_{i=0}^{n} a^{(i)} \mathrm{e}^{\left\langle V_{i}, x\right\rangle}+\sum_{s \in S_{*}} a^{(s)} \mathrm{e}^{\left\langle U_{s}, x\right\rangle} .
$$

Здесь использованы обозначения

$$
a^{(i)}=-\frac{1}{2} d_{i} \xi_{i}, \quad i=0, \ldots, n, \quad a^{(s)}=\frac{1}{2} Q_{s}^{2}, \quad s \in S_{*} .
$$

С математической точки зрения получение точных решений в рассматриваемой $p$ бранной модели сведено к интегрированию уравнений движения лагранжевой системы с $n+l+1$ степенями свободы, описываемой лагранжианом вида

$$
L=\frac{1}{2}\langle\dot{x}, \dot{x}\rangle-\sum_{\mu=1}^{m} a^{(\mu)} \mathrm{e}^{\left\langle b_{\mu}, x\right\rangle},
$$

где $x, b_{\mu} \in \mathbb{R}^{n+l+1}$. Заметим, что кинетическое слагаемое $\langle\dot{x}, \dot{x}\rangle$ не является положительно определенной квадратичной формой, как это обычно имеет место в классической механике. Учитывая псевдоевклидову сигнатуру $(-,+, \ldots,+)$ формы $\langle\cdot, \cdot\rangle$, такие системы можно назвать псевдоевклидовыми системами типа цепочки Тоды, поскольку потенциал, подобный потенциалу в (2.69), определяет хорошо известные в классической механике алгебраические обобщения цепочки Тоды [38]. Проблема интегрируемости псевдоевклидовых систем типа цепочки Тоды изучена в работах [31-36]. Было обнаружено, что условия интегрируемости удобно выражать в терминах характеристических векторов $b_{\mu}$. Далее рассмотрен случай, когда характеристические векторы могут быть интерпретированы как корни алгебры Ли типа $A_{m} \equiv s l(\mathbb{C}, m+1), m \geqslant 2$, по классификации Картана.

\section{3. ИНТЕГРИРОВАНИЕ $p$-БРАННОЙ МОДЕЛИ, СВОДИМОЙ К ОТКРЫТОЙ ЦЕПОЧКЕ ТОДЫ}

Рассмотрим следуюшую систему обыкновенных дифференциальных уравнений:

$$
\ddot{q}^{s}=-\exp \left[\sum_{s^{\prime}=1}^{m} C_{s s^{\prime}} q^{s^{\prime}}\right], \quad s, s^{\prime}=1, \ldots, m
$$


где $\left(C_{s s^{\prime}}\right)$ - невырожденная симметричная матрица. Эти уравнения можно рассматривать как уравнения Эйлера-Лагранжа, полученные из лагранжиана

$$
L=\frac{1}{2} \sum_{s, s^{\prime}=1}^{m} C_{s s^{\prime}} \dot{q}^{s} \dot{q}^{s^{\prime}}-\sum_{s=1}^{m} \exp \left[\sum_{s^{\prime}=1}^{m} C_{s s^{\prime}} q^{s^{\prime}}\right]
$$

Лагранжева система с лагранжианом (3.2) является открытой цепочкой Тоды, ассоциированной с алгеброй Ли типа $A_{m} \equiv s l(m+1, \mathbb{C})$, если матрица $\left(C_{s s^{\prime}}\right)$ представляет собой матрицу Картана этой алгебры, т.е. имеет вид

$$
\left(C_{s s^{\prime}}\right)=\left(\begin{array}{cccccc}
2 & -1 & 0 & \ldots & 0 & 0 \\
-1 & 2 & -1 & \ldots & 0 & 0 \\
0 & -1 & 2 & \ldots & 0 & 0 \\
\ldots & \ldots & \ldots & \ldots & \ldots \\
0 & 0 & 0 & \ldots & 2 & -1 \\
0 & 0 & 0 & \ldots & -1 & 2
\end{array}\right)
$$

Результат интегрирования системы уравнений (3.1) с матрицей (3.3) может быть представлен следуюшим образом [37]:

$$
\mathrm{e}^{-q^{s}(t)} \equiv F_{s}(t)=\sum_{r_{1}<\cdots<r_{s}}^{m+1} v_{r_{1}} \ldots v_{r_{s}} \Delta^{2}\left(r_{1}, \ldots, r_{s}\right) \mathrm{e}^{\left(w_{r_{1}}+\cdots+w_{r_{s}}\right) t}
$$

где $\Delta^{2}\left(r_{1}, \ldots, r_{s}\right)$ обозначает квадрат определителя Вандермонда

$$
\Delta^{2}\left(r_{1}, \ldots, r_{s}\right)=\prod_{r_{i}<r_{j}}\left(w_{r_{i}}-w_{r_{j}}\right)^{2}, \quad \Delta^{2}\left(r_{1}\right) \equiv 1 .
$$

Постоянные $v_{r}$ и $w_{r}$ удовлетворяют условиям

$$
\prod_{r=1}^{m+1} v_{r}=\Delta^{-2}(1, \ldots, m+1), \quad \sum_{r=1}^{m+1} w_{r}=0
$$

Интеграл энергии цепочки Тоды, описываемой этим решением, дается формулой

$$
E=\frac{1}{2} \sum_{s, s^{\prime}=1}^{m} C_{s s^{\prime}} \dot{q}^{s} \dot{q}^{s^{\prime}}+\sum_{s=1}^{m} \exp \left[\sum_{s^{\prime}=1}^{m} C_{s s^{\prime}} q^{s^{\prime}}\right]=\frac{1}{2} \sum_{r=1}^{m+1} w_{r}^{2}
$$


3.1. Модель с риччи-плоскими фактор-пространствами. Рассмотрим случай, когда

$$
\xi_{i}=0, \quad i=0, \ldots, n,
$$

т.е. все фактор-пространства $M_{i}$ являются риччи-плоскими. Тогда задача заключается в том, чтобы проинтегрировать уравнения движения, полученные из лагранжиана

$$
L=\frac{1}{2}\langle\dot{x}, \dot{x}\rangle-\sum_{s \in S_{*}} a^{(s)} \mathrm{e}^{\left\langle U_{s}, x\right\rangle}
$$

при условии нулевой энергии

$$
E=\frac{1}{2}\langle\dot{x}, \dot{x}\rangle+\sum_{s \in S_{*}} a^{(s)} \mathrm{e}^{\left\langle U_{s}, x\right\rangle} \equiv 0 .
$$

Предположим, что характеристические векторы $U_{s} \in \mathbb{R}^{n+l+1}$, которые индуцированы $p$-бранами, удовлетворяют условиям

$$
\begin{aligned}
\left\langle U_{s}, U_{s}\right\rangle & \equiv U^{2}>0, \quad s \in S_{*}, \\
2 \frac{\left\langle U_{s}, U_{s^{\prime}}\right\rangle}{\left\langle U_{s^{\prime}}, U_{s^{\prime}}\right\rangle} & =C_{s s^{\prime}}, \quad s, s^{\prime} \in S_{*},
\end{aligned}
$$

где матрица $\left(C_{s s^{\prime}}\right)$ определена в $(3.3)$. В этом случае векторы $U_{s}$ являются пространственноподобными, линейно независимыми и могут быть интерпретированы как корни алгебры Ли $A_{m} \equiv s l(m+1, \mathbb{C}), m \equiv\left|S_{*}\right|$.

Введем в $\mathbb{R}^{n+l+1}$ базис $\left\{f_{0}, f_{s}, f_{p}\right\}, s=1, \ldots, m, p=m+1, \ldots, n+l$, со следуюшими свойствами:

$$
\begin{gathered}
f_{s}=\frac{2 U_{s}}{\left\langle U_{s}, U_{s}\right\rangle}, \\
\left\langle f_{0}, f_{0}\right\rangle=-1, \quad\left\langle f_{0}, f_{p}\right\rangle=\left\langle f_{0}, f_{s}\right\rangle=0, \\
\left\langle f_{p}, f_{s}\right\rangle=0, \quad\left\langle f_{p}, f_{p^{\prime}}\right\rangle=\delta_{p p^{\prime}} .
\end{gathered}
$$

Заметим, что при $m \equiv\left|S_{*}\right|=n+l$ этот базис не содержит векторы $f_{m+1}, \ldots, f_{n+l}$.

Используя разложение

$$
x(t)=q^{0}(t) f_{0}+\sum_{s=1}^{m}\left[q^{s}(t)-\ln C^{s}\right] f_{s}+q^{m+1}(t) f_{m+1}+\cdots+q^{n+l}(t) f_{n+l},
$$

запишем лагранжиан (3.9) и условие связи (3.10) в виде

$$
L=L_{0}+\frac{2}{U^{2}} L_{A_{m}}+L_{p}, \quad E=E_{0}+\frac{2}{U^{2}} E_{A_{m}}+E_{p} \equiv 0,
$$

где

$$
L_{0}=E_{0}=-\frac{1}{2}\left(\dot{q}^{0}\right)^{2}, \quad L_{p}=E_{p}=\frac{1}{2} \sum_{p=m+1}^{n+l}\left(\dot{q}^{p}\right)^{2}
$$

2 Теоретическая и математическая физика, т. 123, № 3, 2000 г. 
$L_{A_{m}}$ и $E_{A_{m}}$ определены в (3.2) и (3.7), соответственно. Постоянные $C^{s}$ в разложении (3.16) введены следуюшим образом:

$$
C^{s}=\prod_{s^{\prime}=1}^{m}\left(\frac{U^{2}}{2} a^{\left(s^{\prime}\right)}\right)^{C^{s s^{\prime}}}, \quad s=1, \ldots, m,
$$

где $\left(C^{s s^{\prime}}\right)$ - обратная матрица к $\left(C_{s s^{\prime}}\right)$.

Уравнения Эйлера-Лагранжа для координат $q^{0}(t), q^{m+1}(t), \ldots, q^{n+l}(t)$ имеют вид

$$
\ddot{q}^{0}(t)=\ddot{q}^{m+1}(t)=\cdots=\ddot{q}^{n+l}(t)=0 .
$$

Интегрируя их, получим

$$
\begin{aligned}
q^{0}(t) & =a^{0} t+b^{0}, \\
q^{p}(t) & =a^{p} t+b^{p}, \quad p=m+1, \ldots, n+l,
\end{aligned}
$$

где $a^{0}, a^{p}, b^{0}, b^{p}$ - произвольные постоянные. Решение уравнений движения для $q^{1}(t), \ldots, q^{m}(t)$ дается в (3.4). Комбинируя $(3.20),(3.21),(3.4)$ и (3.13), мы представим разложение (3.16) в виде

$$
x(t)=-\frac{1}{U^{2}} \sum_{s=1}^{m} \ln \left[\widetilde{F}_{s}^{2}(t)\right] U_{s}+t Q+P,
$$

где

$$
\widetilde{F}_{s}(t)=C^{s} F_{s}(t), \quad s=1, \ldots, m .
$$

В (3.22) введены следуюшие векторы из $\mathbb{R}^{n+l+1}$ :

$$
\begin{aligned}
& Q=a^{0} f_{0}+\sum_{p=m+1}^{n+l} a^{p} f_{p} \equiv \sum_{A=0}^{n+l} Q^{A} e_{A}, \\
& P=b^{0} f_{0}+\sum_{p=m+1}^{n+l} b^{p} f_{p} \equiv \sum_{A=0}^{n+l} P^{A} e_{A} .
\end{aligned}
$$

Координаты векторов $Q \in \mathbb{R}^{n+l+1}$ и $P \in \mathbb{R}^{n+l+1}$ в каноническом базисе обозначены через $Q^{A}$ и $P^{A}$, соответственно. В силу предположений (3.14), (3.15) они удовлетворяют условиям

$$
\begin{aligned}
& \left\langle Q, U_{s}\right\rangle=\sum_{A, B=0}^{n+l} \bar{G}_{A B} Q^{A} U_{s}^{B}=0, s \in S_{*}, \\
& \left\langle P, U_{s}\right\rangle=\sum_{A, B=0}^{n+l} \bar{G}_{A B} P^{A} U_{s}^{B}=0, s \in S_{*} .
\end{aligned}
$$


Условие нулевой энергии приводится к виду

$$
E=\frac{1}{2} \sum_{A, B=0}^{n+l} \bar{G}_{A B} Q^{A} Q^{B}+\frac{1}{U^{2}} \sum_{r=1}^{m+1} w_{r}^{2}=0 .
$$

Окончательно результат интегрирования рассматриваемой $p$-бранной модели можно представить следующим образом.

1. Функции, определяющие метрику (2.14) в гармонической калибровке времени (2.49), имеют вид

$$
\mathrm{e}^{x^{i}(t)}=\prod_{s=1}^{m}\left[\widetilde{F}_{s}^{2}(t)\right]^{-U_{s}^{i} / U^{2}} \mathrm{e}^{Q^{i} t+P^{i}}, \quad i=0, \ldots, n
$$

2. Дилатонные скалярные поля $\varphi^{\alpha}(u)$ записываются следующим образом:

$$
\varphi^{\alpha}(t)=-\frac{1}{U^{2}} \sum_{s=1}^{m} \ln \left[\widetilde{F}_{s}^{2}(t)\right] U_{s}^{n+\alpha}+t Q^{n+\alpha}+P^{n+\alpha}, \quad \alpha=1, \ldots, l .
$$

3. Скалярные функции $\dot{\Phi}^{s}(t)$, определяюшие $F^{a}$-поля посредством $(2.25),(2.26)$, имеют вид

$$
\dot{\Phi}^{s}(t)=Q_{s} \mathrm{e}^{\left\langle U_{s}, x\right\rangle}=Q_{s} \prod_{s^{\prime}=1}^{m}\left[\widetilde{F}_{s^{\prime}}(t)\right]^{-C_{s s^{\prime}}}, \quad s=1, \ldots, m .
$$

Напомним, что координаты $U_{s}^{A}$, определенные в $(2.52),(2.53)$, должны удовлетворять условиям (3.11), (3.12). Функции $\widetilde{F}_{s}(t)$ определены в $(3.4),(3.19),(3.23)$. Постоянные $v_{r}, w_{r}, Q^{A}, P^{A}$ связаны условиями (3.6),(3.26)-(3.28).

\section{2. Модель с одним фактор-пространством, обладающим ненулевым тен-} зором Риччи. Пусть

$$
\xi_{0} \neq 0, \quad \xi_{1}=\cdots=\xi_{n}=0
$$

т.е. фактор-пространства $M_{1}, \ldots, M_{n}$ являются риччи-плоскими, а $M_{0}$ имеет ненулевой тензор Риччи. В этом случае имеем

$$
\begin{aligned}
& L=\frac{1}{2}\langle\dot{x}, \dot{x}\rangle-a^{(0)} \mathrm{e}^{\left\langle V_{0}, x\right\rangle}-\sum_{s \in S_{*}} a^{(s)} \mathrm{e}^{\left\langle U_{s}, x\right\rangle}, \\
& E=\frac{1}{2}\langle\dot{x}, \dot{x}\rangle+a^{(0)} \mathrm{e}^{\left\langle V_{0}, x\right\rangle}+\sum_{s \in S_{*}} a^{(s)} \mathrm{e}^{\left\langle U_{s}, x\right\rangle} \equiv 0 .
\end{aligned}
$$

Пусть

$$
0 \notin I \quad \forall s=(a, v, I) \in S_{*},
$$

т.е. мировой объем $M_{I}$ каждой $p$-браны с ненулевым электрическим или магнитным зарядом не содержит фактор-пространство $M_{0}$. Легко видеть, что это предположение 
обеспечивает ортогональность индуцированного кривизной вектора $V_{0}$ и любого из векторов $U_{s}$, индуцированного $p$-браной, т.е.

$$
\left\langle V_{0}, U_{s}\right\rangle=0 \quad \forall s \in S_{*} .
$$

Как и ранее, мы требуем, чтобы векторы $U_{s}$ удовлетворяли условиям (3.11), (3.12).

Введем в $\mathbb{R}^{n+l+1}$ базис $\left\{f_{0}, f_{s}, f_{p}\right\}, s=1, \ldots, m, p=m+1, \ldots, n+l$, со следующими свойствами:

$$
\begin{aligned}
f_{0} & =\frac{V_{0}}{\left\langle V_{0}, V_{0}\right\rangle}, \quad f_{s}=\frac{2 U_{s}}{\left\langle U_{s}, U_{s}\right\rangle}, \\
\left\langle f_{p}, V_{0}\right\rangle & =\left\langle f_{p}, f_{s}\right\rangle=0, \quad\left\langle f_{p}, f_{p^{\prime}}\right\rangle=\delta_{p p^{\prime}},
\end{aligned}
$$

где $s=1, \ldots, m, p, p^{\prime}=m+1, \ldots, n+l$. Используя разложение (3.16), мы приводим лагранжиан (3.33) и соответствуюшее условие нулевой энергии (3.34) к виду (3.17) с единственным отличием: в этом случае

$$
L_{0}=\frac{\left(\dot{q}^{0}\right)^{2}}{2\left\langle V_{0}, V_{0}\right\rangle}-a^{(0)} \mathrm{e}^{q^{0}}, \quad E_{0}=\frac{\left(\dot{q}^{0}\right)^{2}}{2\left\langle V_{0}, V_{0}\right\rangle}+a^{(0)} \mathrm{e}^{q^{0}} .
$$

Напомним, что

$$
\left\langle V_{0}, V_{0}\right\rangle=-4 \frac{d_{0}-1}{d_{0}}, \quad a^{(0)}=-\frac{1}{2} d_{0} \xi_{0} .
$$

Как и в предыдушем случае, интегрирование уравнений для $q^{s}$ и $q^{p}$ приводит к (3.4), $(3.21)$. Для $q^{0}(t)$ в этом случае получаем

$$
\mathrm{e}^{-q^{0}(t) / 2}=F_{0}\left(t-t_{0}\right)
$$

где $t_{0}$ - произвольная постоянная. Функция $F_{0}$ определена следуюшим образом:

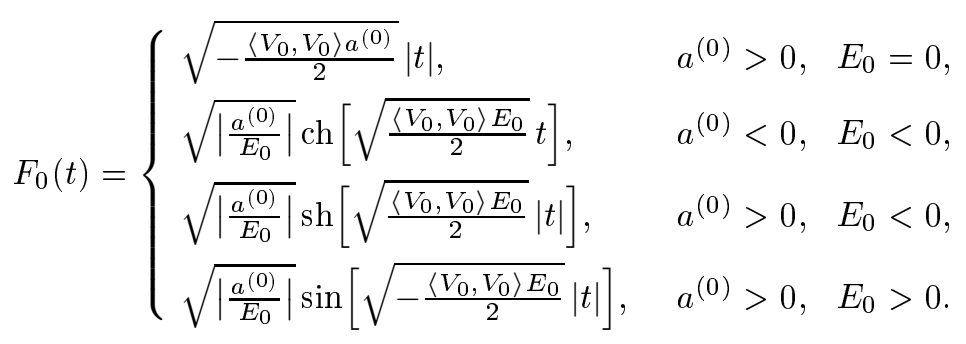

Тогда получаем

$$
x(t)=-\frac{\ln F_{0}^{2}\left(t-t_{0}\right)}{\left\langle V_{0}, V_{0}\right\rangle} V_{0}-\frac{1}{U^{2}} \sum_{s=1}^{m} \ln \left[\widetilde{F}_{s}^{2}(t)\right] U_{s}+t Q+P,
$$

где векторы $Q$ и $P$ вводятся формулами

$$
Q=\sum_{p=m+1}^{n+l} a^{p} f_{p} \equiv \sum_{A=0}^{n+l} Q^{A} e_{A}, \quad P=\sum_{p=m+1}^{n+l} b^{p} f_{p} \equiv \sum_{A=0}^{n+l} P^{A} e_{A} .
$$


В силу предположений (3.38) координаты векторов $Q \in \mathbb{R}^{n+l+1}$ и $P \in \mathbb{R}^{n+l+1}$ в каноническом базисе $\left\{e_{A}\right\}$ удовлетворяют условиям

$$
\begin{aligned}
\left\langle Q, V_{0}\right\rangle & =\sum_{A=0}^{n+l} Q^{A} V_{0, A}=2 \sum_{i=0}^{n} Q^{i}\left(d_{i}-\delta_{i 0}\right)=0 \\
\left\langle P, V_{0}\right\rangle & =\sum_{A=0}^{n+l} P^{A} V_{0, A}=2 \sum_{i=0}^{n} P^{i}\left(d_{i}-\delta_{i 0}\right)=0 \\
\left\langle Q, U_{s}\right\rangle & =\sum_{A=0}^{n+l} Q^{A} U_{s, A}=0, \quad\left\langle P, U_{s}\right\rangle=\sum_{A=0}^{n+l} P^{A} U_{s, A}=0, \quad s=1, \ldots, m .
\end{aligned}
$$

Условие нулевой энергии принимает вид

$$
E=E_{0}+\frac{1}{2} \sum_{A, B=0}^{n+l} \bar{G}_{A B} Q^{A} Q^{B}+\frac{1}{U^{2}} \sum_{r=1}^{m+1} w_{r}^{2}=0 .
$$

Окончательно точное решение может быть представлено следующим образом.

1. Функции, определяюшие метрику (2.14) в гармонической калибровке времени (2.49), имеют вид

$$
\mathrm{e}^{x^{i}(t)}=\left[F_{0}\left(t-t_{0}\right)\right]^{-\delta_{i 0} /\left(d_{0}-1\right)} \prod_{s=1}^{m}\left[\widetilde{F}_{s}^{2}(t)\right]^{-U_{s}^{i} / U^{2}} \mathrm{e}^{Q^{i} t+P^{i}}, \quad i=0, \ldots, n .
$$

2. Дилатонные скалярные поля $\varphi^{\alpha}(u)$ представлены в (3.30).

3. Скалярные функции $\dot{\Phi}^{s}(t)$, определяющие $F^{a}$-поля посредством $(2.25),(2.26)$, выражаются формулами (3.31).

Подчеркнем, что при $m \equiv\left|S_{*}\right|=n+l$ в этом решении нужно положить $Q^{A}=P^{A}=0$, $A=0, \ldots, n+l$. Напомним, что координаты $U_{s}^{A}$, определенные в $(2.52),(2.53)$, должны удовлетворять условиям (3.11), (3.12). Функции $\widetilde{F}_{s}(u)$ определены в $(3.4),(3.19),(3.23)$, функция $F_{0}$ определена в (3.42). Постоянные $v_{r}, w_{r}, Q^{A}, P^{A}, E_{0}$ связаны условиями (3.6), (3.45)-(3.48).

\section{4. ПРИМЕР ИНТЕГРИРУЕМОЙ МОДЕЛИ}

В качестве примера рассмотрим модель на многообразии

$$
\mathbf{M}^{4+n}=\mathbb{R} \times M_{0}^{3} \times M_{1}^{1} \times \cdots \times M_{n}^{1}, \quad n \geqslant 2,
$$

где $M_{0}^{3}$ - внешнее трехмерное пространство постоянной римановой кривизны $K_{0}, M_{i}^{1}$ внутренние пространства размерности 1 . Предположим, что имеется одно дилатонное скалярное поле $\varphi^{1}(t) \equiv \varphi(t)$ и $h_{11}=1 / 2$. Рассмотрим две электрически заряженные 
$(n-1)$-браны с обшим мировым объемом $M_{1}^{1} \times \cdots \times M_{n}^{1}$. Пусть их характеристические векторы имеют вид

$$
\begin{array}{ll}
\left(U_{1}^{A}\right)=2\left(-\frac{n}{n+2}, \frac{2}{n+2}, \cdots, \frac{2}{n+2},-\lambda\right), & \left(U_{1, A}\right)=(0,2, \ldots, 2,-\lambda), \\
\left(U_{2}^{A}\right)=2\left(-\frac{n}{n+2}, \frac{2}{n+2}, \cdots, \frac{2}{n+2}, \lambda\right), & \left(U_{2, A}=(0,2, \ldots, 2, \lambda),\right.
\end{array}
$$

где

$$
f^{-1}(1)=(a, e, I), \quad f^{-1}(2)=(b, e, I), \quad I=\{1, \ldots, n\}, \quad \Delta=\{a, b\} .
$$

Легко проверить, что $U_{1}, U_{2} \in \mathbb{R}^{n+2}$ удовлетворяют условию (3.11) для произвольного $\lambda$. Условие (3.12) также удовлетворяется, если

$$
\lambda= \pm \sqrt{\frac{12 n}{n+2}} .
$$

Следовательно, при условии (4.5) векторы $U_{1}, U_{2}$ могут быть интерпретированы как корни алгебры Ли $A_{2}$. Это означает, что

$$
2 \lambda_{a}(\varphi)=\mp \sqrt{\frac{12 n}{n+2}} \varphi, \quad 2 \lambda_{b}(\varphi)= \pm \sqrt{\frac{12 n}{n+2}} \varphi .
$$

Используя результаты раздела 3 , получаем метрику

$$
\begin{aligned}
g= & \frac{\left[\widetilde{F}_{1}(t) \widetilde{F}_{2}(t)\right]^{1 / 4}}{F_{0}^{3}\left(t-t_{0}\right)}\left(-d t \otimes d t+F_{0}^{2}\left(t-t_{0}\right) g^{(0)}\right)+\left[\widetilde{F}_{1}(t) \widetilde{F}_{2}(t)\right]^{-1 /(2 n)} \times \\
& \times\left(\exp \left\{-2\left(Q^{2}+\cdots+Q^{n}\right) t-2\left(P^{2}+\cdots+P^{n}\right)\right\} g^{(1)}+\right. \\
& \left.+\sum_{k=2}^{n} \exp \left\{2 Q^{k} t+2 P^{k}\right\} g^{(k)}\right),
\end{aligned}
$$

дилатонное скалярное поле

$$
\varphi(t)= \pm \frac{1}{8} \sqrt{\frac{3(n+2)}{n}} \ln \frac{\widetilde{F}_{1}(t)}{\widetilde{F}_{2}(t)}
$$

скалярные функции

$$
\dot{\Phi}^{(a, e, I)}(t)=Q_{(a, e, I)} \frac{\widetilde{F}_{2}(t)}{\widetilde{F}_{1}^{2}(t)}, \quad \dot{\Phi}^{(b, e, I)}(t)=Q_{(b, e, I)} \frac{\widetilde{F}_{1}(t)}{\widetilde{F}_{2}^{2}(t)}
$$


и соответствующие формы

$$
\begin{gathered}
F^{(a, e, I)}=\dot{\Phi}^{(a, e, I)}(t) d t \wedge \tau(I), \quad F^{(b, e, I)}=\dot{\Phi}^{(b, e, I)}(t) d t \wedge \tau(I) \\
\tau(I)=\frac{1}{n !} \sqrt{\prod_{i=1}^{n}\left|g^{(i)}\right| \delta_{M_{1}, \ldots, M_{n}}^{4, \ldots, n+4}} d z^{M_{1}} \wedge \cdots \wedge d z^{M_{n}}
\end{gathered}
$$

Использованы следующие обозначения:

$$
\begin{aligned}
& F_{0}(t)= \begin{cases}\exp \left[2 \delta \sqrt{-\frac{E_{0}}{3}} t\right], & K_{0}=0, \quad \delta^{2}=1, \\
\sqrt{\left|\frac{3 K_{0}}{E_{0}}\right|} \operatorname{ch}\left[2 \sqrt{-\frac{E_{0}}{3}} t\right], & K_{0}>0, \\
\sqrt{\left|\frac{3 K_{0}}{E_{0}}\right|} \operatorname{sh}\left[2 \sqrt{-\frac{E_{0}}{3}}|t|\right], & K_{0}<0,\end{cases} \\
& \widetilde{F}_{1}(t)=\frac{8 n}{n+2} Q_{(a, e, I)}^{4 / 3} Q_{(b, e, I)}^{2 / 3} \sum_{i=1}^{3} v_{i} \mathrm{e}^{w_{i} t} \\
& \widetilde{F}_{2}(t)=\frac{8 n}{n+2} Q_{(a, e, I)}^{2 / 3} Q_{(b, e, I)}^{4 / 3} \sum_{i=1}^{3} u_{i} \mathrm{e}^{-w_{i} t},
\end{aligned}
$$

где $Q^{2}, \ldots, Q^{n}, P^{2}, \ldots, P^{n}, t_{0}$ - произвольные постоянные, $Q_{(a, e, I)}, Q_{(b, e, I)}$ - ненулевые постоянные. Постоянные $w_{1}, w_{2}, w_{3}$ удовлетворяют условию

$$
w_{1}+w_{2}+w_{3}=0
$$

a $v_{1}, v_{2}, v_{3}$ - условию

$$
v_{1} v_{2} v_{3}=\left[\left(w_{1}-w_{2}\right)\left(w_{1}-w_{3}\right)\left(w_{2}-w_{3}\right)\right]^{-2}
$$

Постоянные $u_{1}, u_{2}, u_{3}$ определяются формулой

$$
u_{1}=\left(w_{2}-w_{3}\right)^{2} v_{2} v_{3}
$$

и ее циклическими перестановками. Постоянная $E_{0}$ дается формулой

$$
-E_{0}=\frac{1}{2} \sum_{i, j=2}^{n}\left(\delta_{i j}+1\right) Q^{i} Q^{j}+\frac{n+2}{32 n}\left[w_{1}^{2}+w_{2}^{2}+w_{3}^{2}\right]>0 .
$$

Данное точное решение содержит, как и требуется, $2 n+5$ независимых постоянных.

При исследовании эволюции многомерной Вселенной, описываемой метрикой (2.14), возникает проблема, связанная с неоднозначностью конформного множителя $\Omega$ в соотношении

$$
{ }^{(4)} g=\Omega\left(-\mathrm{e}^{2 \gamma_{0}(t)} d t \otimes d t+\mathrm{e}^{2 x^{0}(t)} g^{(0)}\right)
$$


меж ду физической четырехмерной метрикой ${ }^{(4)} g$ и четырехмерным блоком в $D$-мерной метрике (здесь мы рассматриваем $M_{0}$ как внешнее трехмерное пространство). Конформный множитель $\Omega$ определяет видгравитационного сектора действия, полученного из (2.1) посредством размерной редукции. Выбор конформного множителя $\Omega$ называется конформной калибровкой (см., например, [39, 40]). Если положить, например,

$$
\Omega=\Omega_{\mathrm{E}}=\left(\prod_{i=1}^{n} \mathrm{e}^{d_{i} x^{i}(t)}\right)^{2 /\left(d_{0}-1\right)}, d_{0}=3
$$

то гравитационный сектор действия (2.1) после размерной редукции имеет эйнштейновский вид по отношению к метрике ${ }^{(4)} g[39]$, т.е.

$$
S_{\mathrm{E}}^{\text {grav }}=\int_{\mathbf{M}^{4}} d^{4} z \sqrt{|(4) g|} R\left[^{(4)} g\right], \quad \mathbf{M}^{4}=\mathbb{R} \times M_{0} .
$$

Поэтому конформная калибровка (4.20) называется эйнштейновской. Если в (4.19) положить

$$
\Omega=\Omega_{\mathrm{BD}} \equiv 1,
$$

то получим

$$
S_{\mathrm{BD}}^{\mathrm{grav}}=\int_{\mathrm{M}^{4}} d^{4} z \sqrt{\left|{ }^{(4)} g\right|} \Omega_{\mathrm{E}} R\left[^{(4)} g\right] .
$$

Благодаря множителю $\Omega_{\mathrm{E}}$ четырехмерная теория с действием (4.23) схожа с теорией Бранса-Дикке, поэтому такая конформная калибровка называется калибровкой Бранса-Дикке. Проблема определения, какая из калибровок наиболее предпочтительна с точки зрения физики, обсуждается в работе [40].

Приступим к анализу метрики (4.7). По построению функции (4.13), (4.14) гармонического времени $t$ положительны всюду на некотором интервале $(a, b)$. Легко показать, что только для положительных постоянных $v_{1}, v_{2}, v_{3}$ существует интервал $(a, b)$ такой, что $\widetilde{F}_{1}(t)>0, \widetilde{F}_{2}(t)>0 \forall t \in(a, b) ; a=-\infty, b=+\infty$ для $K_{0} \geqslant 0$ и $a=t_{0}, b=+\infty$ (или $a=-\infty, b=t_{0}$ ) для $K_{0}<0$. Кроме того, справедлива следуюшая асимптотика:

$$
\widetilde{F}_{1}(t) \widetilde{F}_{2}(t) \sim \operatorname{ch}[(W-w) t], \quad t \rightarrow \pm \infty,
$$

где

$$
W=\max \left\{w_{1}, w_{2}, w_{3}\right\}>0, \quad w=\min \left\{w_{1}, w_{2}, w_{3}\right\}<0 .
$$

Это приводит к тому, что если постоянные $Q^{2}, \ldots, Q^{n}$ достаточно малы, то масштабные факторы всех внутренних пространств $M_{1}, \ldots, M_{n}$ стремятся к нулю при $t \rightarrow \pm \infty$. Например, такое свойство имеет место для изотропного внутреннего пространства $M_{1} \times \cdots \times M_{n}$, если $Q^{2}=\cdots=Q^{n}=0$. Подобная эволюция внутренних пространств соответствует ненаблюдаемости дополнительных измерений на современной стадии и является привлекательной с точки зрения физики. 
Масштабньй фактор внешнего пространства $M_{0}^{3}$ и синхронное время $\tau$ зависят от конформной калибровки. Рассмотрим, например, эйнштейновскую конформную калибровку. В этом случае масштабный фактор внешнего пространства имеет вид

$$
a_{M_{0}}^{(\mathrm{E})}=\frac{1}{\sqrt{F_{0}\left(t-t_{0}\right)}},
$$

а синхронное время $\tau$ определяется уравнением

$$
F_{0}^{3 / 2}\left(t-t_{0}\right) d \tau=d t
$$

Нетрудно показать, что $a_{M_{0}}^{(\mathrm{E})}$ удовлетворяет уравнению

$$
\frac{d a_{M_{0}}^{(\mathrm{E})}}{d \tau}+K_{0}=-\frac{E_{0} / 3}{\left[a_{M_{0}}^{(\mathrm{E})}\right]^{4}},
$$

которое совпадает с уравнением для масштабного фактора в четырехмерной модели $\Phi$ ридмана с предельно жесткой материей. Следовательно, в эйнштейновской конформной калибровке трехмерная часть многомерной Вселенной эволюционирует так же, как Вселенная Фридмана, заполненная предельно жесткой материей.

Таким образом, рассмотренный пример показывает наличие привлекательных с точки зрения физики решений для $p$-бранных космологических моделей, ассоциированных с алгебрами Ли типа $A_{m}$.

Благодарности. Авторы благодарят Российский фонд фундаментальных исследований за финансовую поддержку (проект № 98-02-16414).

\section{Список литературы}

[1] K. S. Stelle. Lectures on supergravity $p$-branes. hep-th $/ 9701088$.

[2] I. Ya. Aref'eva, O. A. Rytchkov. Incidence matrix description of intersecting $p$-brane solutions. hep-th/9612236

[3] I. Ya. Aref'eva, M. G. Ivanov, O. A. Rytchkov. Properties of intersecting $p$-branes in various dimensions. hep-th/9702077.

[4] V. D. Ivashchuk, V. N. Melnikov. Gravit. Cosmology. 1996. V. 2. P. 297; hep-th/9612089.

[5] V. D. Ivashchuk, V. N. Melnikov. Phys. Lett. B. 1997. V. 403. P. 23.

[6] H. Lü, S. Mukherji, C.N. Pope, K.-W. Xu. Cosmological solutions in string theories. hep-th/9610107.

[7] H. Lü, C. N. Pope, K.-W. Xu. Liouville and Toda solitons in M-theory. hep-th/9604058.

[8] M. Cvetic, A. A. Tseytlin. Nucl. Phys. B. 1996. V. 478. P. 181.

[9] V. D. Ivashchuk, V. N. Melnikov. Class. Q. Grav. 1997. V. 14. P. 3001; hep-th/9705036.

[10] K.A. Bronnikov, M.A. Grebeniuk, V.D. Ivashchuk, V.N. Melnikov. Gravit. Cosmology. 1997. V. 3. P. 105.

[11] M. A. Grebeniuk, V.D. Ivashchuk, V. N. Melnikov. Gravit. Cosmology. 1997. V. 3. P. 243; gr-qc/9708031.

[12] K. A. Bronnikov, V. D. Ivashchuk, V. N. Melnikov. Gravit. Cosmology. 1997. V. 3. P. 203; gr-qc/9710054. 
[13] K. A. Bronnikov, U. Kasper, M. Rainer. Intersecting electric and magnetic $p$-branes: spherically symmetric solutions. gr-qc/9708058.

[14] K. A. Bronnikov. Gravit. Cosmology. 1998. V. 4. P. 49; hep-th/9710207.

[15] I. Ya. Aref'eva, M. G. Ivanov, I. V. Volovich. Non-extremal intersecting $p$-branes in various dimensions. hep-th/9702079.

[16] N. Ohta. Intersection rules for non-extreme $p$-branes. hep-th/9702164.

[17] V.D. Ivashchuk, V. N. Melnikov. J. Math. Phys. 1998. V. 39. P. 2866; hep-th/9708157.

[18] V. D. Ivashchuk, V. N. Melnikov. Class. Q. Grav. 1999. V. 16. P. 849; hep-th/9802121.

[19] V. D. Ivashchuk, S.-W. Kim, V. N. Melnikov. J. Math Phys. 1999. V. 40. № 8. P. 4072; hep-th/9803006.

[20] M. J. Duff, R. R. Khuri, J. X. Lu. Phys. Rep. 1995. V. 259. P. 213.

[21] N. Khvengia, Z. Khvengia, H. Lü, C. N. Pope. Toward field theory of F-theory. hep-th/9703012.

[22] J. M. Schwarz. Lectures on superstring and M-theory dualities. hep-th/9607201.

[23] M. J. Duff. M-theory (the theory formerly known as strings). hep-th/9608117.

[24] C. Hull, P. Townsend. Nucl. Phys. B. 1995. V. 438. P. 109.

[25] P. Horava, E. Witten. Nucl. Phys. B. 1996. V. 460. P. 506.

[26] C. Vafa. Nucl. Phys. B. 1996. V. 469. P. 403.

[27] C. M. Hull. Nucl. Phys. B. 1996. V. 468. P. 113.

[28] E. Cremmer, B. Julia, J. Scherk. Phys. Lett. B. 1978. V. 76. P. 409.

[29] V.D. Ivashchuk, V. N. Melnikov, A. I. Zhuk. Nuovo Cimento B. 1989. V. 104. P. 575.

[30] U. Bleyer, V. D. Ivashchuk, V. N. Melnikov, A. I. Zhuk. Nucl. Phys. B. 1994. V. 429. P. 177.

[31] V. D. Ivashchuk, V. N. Melnikov. Int. J. Mod. Phys. D. 1994. V. 3. P. 795.

[32] V. D. Ivashchuk, V. N. Melnikov. Gravit. Cosmology. 1995. V. 1. P. 204.

[33] V.D. Ivashchuk, V. N. Melnikov. Class. Q. Grav. 1995. V. 12. P. 809.

[34] V.R. Gavrilov, V.D. Ivashchuk, V. N. Melnikov. J. Math. Phys. 1995. V. 36. P. 5829.

[35] В.Р. Гаврилов, В.Н. Мельников. ТМФ. 1998. Т. 114. С. 454.

[36] V.R. Gavrilov, U. Kasper, V. N. Melnikov, M. Rainer. Gen. Relat. Gravit. 1999. V. 31. P. 139.

[37] A. Anderson. J. Math. Phys. 1996. V. 37. P. 1349.

[38] M. Тода. Теория нелинейных решеток. М.: Мир, 1984.

[39] U. Gunter, A. Zhuk. Phys. Rev. D. 1997. V. 56. P. 6391.

[40] G. Magnano, M. Sokolowski. Phys. Rev. D. 1994. V. 50. P. 5039.

Поступила в редакцию 25.V.1999 г., после доработки 5.XI.1999 г. 